\title{
How tight is the corner relaxation? Insights gained from the stable set problem
}

\author{
Gérard Cornuéjols $^{\mathrm{a}, 1}$, Carla Michini ${ }^{\mathrm{b}, 2, *}$, Giacomo Nannicini ${ }^{\mathrm{c}, 3}$ \\ ${ }^{a}$ Tepper School of Business, Carnegie Mellon University, Pittsburgh, PA. \\ ${ }^{b}$ Dipartimento di Ingegneria Informatica Automatica e Gestionale Antonio Ruberti, \\ Sapienza Università di Roma, Roma, Italy. \\ ${ }^{c}$ Singapore University of Technology and Design, Singapore and MIT Sloan School of \\ Management, Cambridge, MA.
}

\begin{abstract}
The corner relaxation of a mixed-integer linear program is a central concept in cutting plane theory. In a recent paper Fischetti and Monaci provide an empirical assessment of the strength of the corner and other related relaxations on benchmark problems. In this paper we give a precise characterization of the bounds given by these relaxations for the edge formulation of the maximum stable set problem in a graph.
\end{abstract}

Keywords: Stable set, corner relaxation, cutting planes

\section{Motivation}

Consider a Mixed-Integer Linear Program (MILP) in standard form:

$$
\min \left\{c x: A x=b, x \in \mathbb{Z}_{+}^{n_{1}} \times \mathbb{R}_{+}^{n_{2}}\right\}
$$

\footnotetext{
${ }^{*}$ Corresponding author

Email addresses: gc0v@andrew.cmu.edu (Gérard Cornuéjols), michini@dis. uniroma1.it (Carla Michini), nannicini@sutd.edu.sg (Giacomo Nannicini)

${ }^{1}$ Supported by NSF grant CMMI1024554 and ONR grant N00014-12-1-0032.

${ }^{2}$ Partially supported by Fondazione Ugo Bordoni.

${ }^{3}$ Supported by SUTD grant SRES11012. Part of this work was conducted under support of an IBM fellowship.
} 


\section{Report Documentation Page}

Form Approved

OMB No. 0704-0188

Public reporting burden for the collection of information is estimated to average 1 hour per response, including the time for reviewing instructions, searching existing data sources, gathering and maintaining the data needed, and completing and reviewing the collection of information. Send comments regarding this burden estimate or any other aspect of this collection of information,

including suggestions for reducing this burden, to Washington Headquarters Services, Directorate for Information Operations and Reports, 1215 Jefferson Davis Highway, Suite 1204, Arlington

VA 22202-4302. Respondents should be aware that notwithstanding any other provision of law, no person shall be subject to a penalty for failing to comply with a collection of information if it

does not display a currently valid OMB control number.

\begin{tabular}{|c|c|c|}
\hline $\begin{array}{l}\text { 1. REPORT DATE } \\
12 \text { FEB } 2012\end{array}$ & 2. REPORT TYPE & $\begin{array}{l}\text { 3. DATES COVERED } \\
\mathbf{0 0 - 0 0 - 2 0 1 2} \text { to 00-00-2012 }\end{array}$ \\
\hline \multirow{3}{*}{\multicolumn{2}{|c|}{$\begin{array}{l}\text { 4. TITLE AND SUBTITLE } \\
\text { pow tight is the corner relaxation? Insights gained from the stable set } \\
\text { problem }\end{array}$}} & 5a. CONTRACT NUMBER \\
\hline & & 5b. GRANT NUMBER \\
\hline & & 5c. PROGRAM ELEMENT NUMBER \\
\hline \multirow{3}{*}{\multicolumn{2}{|c|}{ 6. AUTHOR(S) }} & 5d. PROJECT NUMBER \\
\hline & & 5e. TASK NUMBER \\
\hline & & 5f. WORK UNIT NUMBER \\
\hline \multicolumn{2}{|c|}{$\begin{array}{l}\text { 7. PERFORMING ORGANIZATION NAME(S) AND ADDRESS(ES) } \\
\text { Carnegie Mellon University,Tepper School of } \\
\text { Business,Pittsburgh,PA,15213 }\end{array}$} & $\begin{array}{l}\text { 8. PERFORMING ORGANIZATION } \\
\text { REPORT NUMBER }\end{array}$ \\
\hline \multirow{2}{*}{\multicolumn{2}{|c|}{ 9. SPONSORING/MONITORING AGENCY NAME(S) AND ADDRESS(ES) }} & 10. SPONSOR/MONITOR'S ACRONYM(S) \\
\hline & & $\begin{array}{l}\text { 11. SPONSOR/MONITOR'S REPORT } \\
\text { NUMBER(S) }\end{array}$ \\
\hline
\end{tabular}

12. DISTRIBUTION/AVAILABILITY STATEMENT

Approved for public release; distribution unlimited

13. SUPPLEMENTARY NOTES

14. ABSTRACT

The corner relaxation of a mixed-integer linear program is a central concept in cutting plane theory. In a recent paper Fischetti and Monaci provide an empirical assessment of the strength of the corner and other related relaxations on benchmark problems. In this paper we give a precise characterization of the bounds given by these relaxations for the edge formulation of the maximum stable set problem in a graph.

15. SUBJECT TERMS

16. SECURITY CLASSIFICATION OF:

\begin{tabular}{c|c|c|c|c|c}
$\begin{array}{c}\text { a. REPORT } \\
\text { unclassified }\end{array}$ & $\begin{array}{c}\text { b. ABSTRACT } \\
\text { unclassified }\end{array}$ & $\begin{array}{c}\text { ABSTRACT } \\
\text { c. THIS PAGE } \\
\text { unclassified }\end{array}$ & $\begin{array}{c}\text { OF PAGES } \\
\text { Report (SAR) }\end{array}$ & $\mathbf{2 7}$ & RESPONSIBLE PERSON \\
& & &
\end{tabular}


where $c \in \mathbb{Q}^{n_{1}+n_{2}}, A \in \mathbb{Q}^{m \times\left(n_{1}+n_{2}\right)}, b \in \mathbb{Q}^{m}$. Its Linear Programming (LP) relaxation is the problem:

$$
\min \left\{c x: A x=b, x \in \mathbb{R}_{+}^{n_{1}+n_{2}}\right\} .
$$

An exact solution method for (MILP) is Branch-and-Cut. One of the components of Branch-and-Cut is the generation of cutting planes valid for (MILP), which are then added to $\left(\mathrm{LP}^{\prime}\right)$. Most general purpose cutting planes, such as Gomory mixed-integer [1] and mixed-integer rounding [2] cuts, are valid for the corner relaxation of (MILP), introduced by Gomory [3]. Studying the strength of the corner relaxation is therefore of both theoretical and practical interest. Given a basis $B$ of $\left(\mathrm{LP}^{\prime}\right)$, the corner relaxation is the convex hull of the integer points of the problem obtained from (MILP) by dropping nonnegativity on the basic variables. If nonnegativity is dropped on the strictly positive basic variables only, we call the convex hull of the resulting set of points strict corner relaxation (there is no standard terminology for this relaxation in the literature). If ( $\left.\mathrm{LP}^{\prime}\right)$ has primal degeneracy, the strict corner relaxation can be stronger than the corner relaxation.

Fischetti and Monaci [4] empirically study the strength of the corner relaxation, strict corner relaxation and other related polyhedra on a set of benchmark MILP instances. They compare the objective value of the integer optimum of (MILP) with the bounds given by the strict corner relaxation, the corner relaxation associated with an optimal basis of $\left(\mathrm{LP}^{\prime}\right),\left(\mathrm{LP}^{\prime}\right)$ alone, and $\left(\mathrm{LP}^{\prime}\right)$ strengthened by one round of cutting planes from an optimal basis. They conclude that:

- For problems with binary variables, the corner relaxation is often a weak approximation of (MILP).

- The strict corner relaxation gives on average $50 \%$ better bounds (in relative terms) than the corner relaxation.

- The conclusion that the corner relaxation is often a weak approximation of (MILP) is mitigated by the fact that, in practice, cutting planes are added to $\left(\mathrm{LP}^{\prime}\right)$ and this often gives much better bounds.

In this paper we study the relaxations discussed by Fischetti and Monaci in the particular case of the edge formulation of the stable set problem. This is an important combinatorial optimization problem, and stable set type constraints appear in the MILP formulation of many real-world problems. 
We give a precise characterization of the bounds studied by Fischetti and Monaci for this particular combinatorial problem. The reason for choosing this problem is that it is one of the very few where the structure of the bases is well understood, allowing a tight analysis of the relaxations.

Given a graph with $n$ vertices it is known that, under mild assumptions, $\left(\mathrm{LP}^{\prime}\right)$ of the edge formulation for the maximum stable set problem has value $n / 2$. For the most common random graph models and for $n \rightarrow \infty$, these assumptions hold with probability $1[5,6]$. The results proven in this paper can be summarized as follows. We show that, for a connected graph $G$, if the graph admits a perfect matching or a nearly perfect matching, there exists an optimal basis $B$ of $\left(\mathrm{LP}^{\prime}\right)$ such that the associated corner relaxation gives a bound of $\lfloor n / 2\rfloor$. If the vertices of the graph can be partitioned into cliques of size at least 3 , the split closure [7] yields a bound of $n / 3$. If all cliques in the partition have size 3 , the same bound can also be obtained from a corner relaxation associated with an optimal basis. We show that in some cases, generating cutting planes from a corner relaxation and adding them to ( $\left.\mathrm{LP}^{\prime}\right)$ significantly improves the corner relaxation bound. Finally, we show that the strict corner relaxation yields the optimal value of (MILP).

Our results confirm the empirical study of Fischetti and Monaci [4]. They lead to the following observations. The corner relaxation can be a very weak approximation of the integer hull. Using cuts from multiple bases of ( $\left.\mathrm{LP}^{\prime}\right)$ can greatly improve over using a single basis; for this line of research, see e.g. [8]. Degeneracy of $\left(\mathrm{LP}^{\prime}\right)$ plays a major role. The stable set problem is highly degenerate, and the difference in the bounds given by corner relaxations from two different optimal bases can be arbitrarily large. Furthermore, the strict corner relaxation can be much stronger than corner relaxations. Although generating cutting planes from the strict corner relaxation is difficult, this is another indication that, in the presence of LP degeneracy, exploiting multiple degenerate bases for cut generation could give significantly better bounds than working with just a single basis. Finally, the strength of the corner relaxation is not always a good indicator of the strength of the cutting planes that can be obtained from it, when these cuts are added to the LP relaxation.

\section{Our Main Results}

Consider a simple graph $G(V, E)$, where $V$ and $E$ are the sets of $n$ vertices and $m$ edges of $G$, respectively. A stable set (vertex packing, independent set, anticlique) of $\mathrm{G}$ is a set of pairwise non-adjacent vertices. It follows that a 
vector $x \in\{0,1\}^{n}$ is the incidence vector of a stable set of $G$ if and only if it satifies $x_{u}+x_{v} \leq 1$, for all $(u, v) \in E$.

The problem of finding a maximum stable set in a graph can be consequently formulated as the integer program:

$$
\begin{array}{rll}
z_{\mathrm{STAB}}=\max & \sum_{i=1}^{n} x_{i} & \\
\text { s.t. } & x_{i}+x_{j}+y_{i j}=1 & \\
& y_{i j} \geq 0 & \forall(i, j) \in E \\
& x_{i} \in \mathbb{Z}_{+}^{n} & \forall i \in j) \in E
\end{array}
$$

The stable set polytope, denoted by $S(G)$, is the convex hull of the incidence vectors of stable sets, which correspond to feasible solutions of (STAB). For the MILP (STAB), the LP relaxation has the form

$$
\begin{array}{rll}
z_{\mathrm{LP}}=\max & \sum_{i=1}^{n} x_{i} & \\
\text { s.t. } & x_{i}+x_{j}+y_{i j}=1 & \forall(i, j) \in E \\
& x_{i} \geq 0 & \forall i \in V \\
& y_{i j} \geq 0 & \forall(i, j) \in E,
\end{array}
$$

whose feasible set will be denoted $L P(G)$.

Let $A$ denote the edge-vertex incidence matrix of $G$. Let $\mathcal{B}$ stand for the set of all bases of the constraint matrix $\left[\begin{array}{ll}A & I\end{array}\right]$. Note that the rows of (LP) are linearly independent, therefore a basis consists of $m$ columns. We denote by $B$ an element of $\mathcal{B}$ and by $N$ the resulting nonbasic submatrix. To avoid heavy notation, we may also use $B$ and $N$ to denote the corresponding sets of indices. The variables can be partitioned according to each basis $B \in \mathcal{B}$ as $x=\left(x_{B}, x_{N}\right)^{T}$ and $y=\left(y_{B}, y_{N}\right)^{T}$. Discarding nonnegativity constraints on the basic variables, we get a relaxation of (STAB). The convex hull of the resulting set of feasible solutions is the so-called corner polyhedron associated with basis $B$, denoted in the remainder by corner $(B)$. If the basic solution associated with basis $B$ is not integral, then it does not belong to corner $(B)$, and a valid inequality for $\operatorname{corner}(B)$ can be generated, such that the fractional solution is cut off. It has been shown [9] that all valid inequalities necessary to describe corner $(B)$ can be derived from one row of the simplex tableau 
associated to basis $B$ as Chvátal-Gomory cuts. Define now the intersection closure as the intersection of the corner polyhedra associated to all bases and denote it by $\operatorname{int}(\mathcal{B})$, namely

$$
\operatorname{int}(\mathcal{B})=\bigcap_{B \in \mathcal{B}} \operatorname{corner}(B)
$$

It has been proven [9] that, for the stable set formulation (STAB), the set $\operatorname{int}(\mathcal{B})$ and the split, Chvátal and $\left\{0, \frac{1}{2}\right\}$-Chvátal closures are all identical.

We address two additional relaxations of (STAB). The first one, that we call strict corner relaxation, is obtained from (STAB) by relaxing nonnegativity constraints on those variables that are strictly positive in an optimal solution $x^{*}$ of (LP). The convex hull of the feasible points of the strict corner relaxation is the strict corner polyhedron, denoted by strict $\left(x^{*}\right)$. The second relaxation is defined by intersecting $\operatorname{corner}(B)$ and $L P(G)$ for a given $B \in \mathcal{B}$, and we denote it by $\mathrm{LP} \cap \operatorname{corner}(B)$. The reason for studying this relaxation is that $\mathrm{LP} \cap \operatorname{corner}(B)$ corresponds to strengthening (LP) with cutting planes valid for corner $(B)$, and is therefore highly relevant in practice.

In this paper, we estimate and compare the bounds obtained by optimizing over corner $(B), \operatorname{int}(\mathcal{B}), \operatorname{strict}\left(x^{*}\right)$ and LP $\cap$ corner $(B)$. In other words, we study the following problems:

$$
\begin{aligned}
z_{\text {corner }(B)} & =\max \left\{\mathbf{1}^{T} x: x \in \operatorname{corner}(B)\right\}, & (\operatorname{corner}(B)) \\
z_{\text {int }(\mathcal{B})} & =\max \left\{\mathbf{1}^{T} x: x \in \operatorname{int}(\mathcal{B})\right\}, & (\operatorname{int}(\mathcal{B})) \\
z_{\text {strict }\left(x^{*}\right)} & =\max \left\{\mathbf{1}^{T} x: x \in \operatorname{strict}\left(x^{*}\right)\right\}, & \left(\operatorname{strict}\left(x^{*}\right)\right) \\
z_{\mathrm{LP} \cap \operatorname{corner}(B)} & =\max \left\{\mathbf{1}^{T} x: x \in L P(G) \cap \operatorname{corner}(B)\right\} . & (\operatorname{LP} \cap \operatorname{corner}(B))
\end{aligned}
$$

It is a well-known result that, in basic feasible solutions to (LP), variables must be $\left(0, \frac{1}{2}, 1\right)$-valued $[10,11,12]$. For an optimal solution $x^{*}$ to (LP), we define $P=\left\{i: x_{i}^{*}=1\right\}, Q=\left\{i: x_{i}^{*}=\frac{1}{2}\right\}, p=|P|$ and $q=|Q|$. Therefore $z_{\mathrm{LP}}=p+\frac{q}{2}$. Define $G[Q]$ as the subgraph of $G$ induced by vertices of $Q$.

Our main results are stated in the following theorems.

Theorem 2.1. If $G[Q]$ is connected and admits a perfect or nearly perfect matching, then there exists an optimal basis $B$ associated to $x^{*}$ such that $z_{\text {corner }(B)}=p+\left\lfloor\frac{q}{2}\right\rfloor$. 
Theorem 2.2. Optimizing over strict $\left(x^{*}\right)$ yields the same optimal value as optimizing over the original integer problem, namely $z_{\text {strict }\left(x^{*}\right)}=z_{S T A B}$.

Theorem 2.3. If the vertices of $Q$ can be partitioned into cliques of size at least $3, z_{\text {int }(\mathcal{B})}=p+\frac{q}{3}$. If all cliques of the partition have size exactly 3 , there exists an optimal basis $B$ associated to $x^{*}$ such that $z_{\text {corner }(B)}=p+\frac{q}{3}$.

Theorem 2.4. For an optimal basis $B$ associated to $x^{*}$, the difference between $z_{\text {corner(B) }}$ and $z_{L P \cap \operatorname{corner}(B)}$ is at most $\frac{q}{8}$, and there are graphs for which this bound is tight.

\section{Complete description of the corner polyhedron and of the inter- section closure}

In this section, we describe the structure of the bases of (LP) and present results from $[9]$ that will be used in the proofs.

Let $B \in \mathcal{B}$ be a basis, feasible or infeasible. Let $V_{B}$ and $V_{N}$ represent the set of basic and nonbasic vertices, indexing variables $x_{B}$ and $x_{N}$, respectively. Similarly, partition edges into $E_{B}$ and $E_{N}$. In order to characterize the structure of the basis, consider $G_{B}$, which is obtained from $G$ by removing the basic edges. Let $C_{i}\left(V_{i}, E_{i}\right), i=1, \ldots, k$ be the connected components of $G_{B}$ that are not defined by a single vertex.

A graph $G(V, E)$ is called a 1 -tree if it is connected and $|E|=|V|$. A 1-tree contains a unique cycle. Define $I_{0}$ and $I_{1}$ as the subsets of $\{1, \ldots, k\}$ indexing tree and 1-tree components, respectively.

Theorem 3.1. [9] For every $B \in \mathcal{B}$, each connected component of $G_{B}$ is either a tree or a 1-tree with an odd cycle. Each tree has exactly one nonbasic vertex. The vertices of every 1-tree are all basic.

Given $B \in \mathcal{B}$, for all $i=1, \ldots, k$ denote by $B_{i}$ the submatrix of $B$ defined by the rows and columns indexed by $E_{i}$ and $V_{i} \cap B$, respectively.

Lemma 3.1. [13] Given $B \in \mathcal{B}$, for $i \in I_{0}, B_{i}^{-1} \mathbf{1} \in\{0,1\}^{\left|V_{i}\right|}$. For $i \in I_{1}$, $B_{i}^{-1} \mathbf{1}=\left(\frac{1}{2}\right) \mathbf{1}$.

Moreover, it is possible to state the converse of Theorem 3.1.

Theorem 3.2. Let $G_{B}\left(V, E_{N}\right)$ be a subgraph of $G$, whose connected components are isolated vertices, trees and 1-trees with an odd cycle. Let $S$ be 
the set of isolated vertices of $G_{B}$, and denote by $C_{i}\left(V_{i}, E_{i}\right), i=1, \ldots, k$ the remaining connected components. Let $I_{0} \subseteq\{1, \ldots, k\}$ index the tree components of $G_{B}$ and, for $i \in I_{0}$, let $v_{i}$ be an arbitrarily chosen vertex of the tree component $C_{i}$. Define $V_{N}=\left(\bigcup_{i \in I_{0}} v_{i}\right) \cup S, V_{B}=V \backslash V_{N}, E_{B}=E \backslash E_{N}$. Then $B=V_{B} \cup E_{B}$ is a basis of $L P(G)$.

Proof. First, let us group the equations of (LP) according to the edges of $E_{N}$ and $E_{B}$ respectively, to get

$$
\begin{aligned}
& \bar{A} x+y_{N}=1, \\
& \hat{A} x+y_{B}=1,
\end{aligned}
$$

where $A=\left[\begin{array}{c}\bar{A} \\ \hat{A}\end{array}\right]$. Notice that $\bar{A}$ is the edge-vertex incidence matrix of $G_{B}$. Precisely, if $A_{i}$ is the $\left|E_{i}\right| \times\left|V_{i}\right|$ incidence matrix of $C_{i}$, then $\bar{A}$ can be organized as

$$
\bar{A}=\left[\begin{array}{llll|l}
A_{1} & & & & \\
& A_{2} & & & 0 \\
& & \ddots & & \\
& & & A_{k} &
\end{array}\right] .
$$

Remark that the last zero columns correspond to those components of $G_{B}$ which consist of single vertices.

For every tree component $C_{i}, i \in I_{0}$, let us partition $A_{i}=\left[B_{i} N_{i}\right]$, where $N_{i}$ consists of a single column, which is the one indexed by vertex $v_{i}$. It is easy to check that each matrix $B_{i}$ is square and invertible, because it can be expressed as a triangular matrix, by reordering the vertices of the tree from the leaves towards the root $v_{i}$.

For every 1-tree component $C_{i}, i \in I_{1}$, define $B_{i}=A_{i}$. Also in this case each matrix $B_{i}$ is square and invertible, as it can be expressed as a block matrix of the form

$$
B_{i}=\left[\begin{array}{cc}
T_{i} & D_{i} \\
0 & K_{i}
\end{array}\right]
$$

where $K_{i}$ is the edge-vertex incidence matrix of the odd cycle and $T_{i}$, together with an extra column of $D_{i}$, is the edge-vertex incidence matrix of the acyclic part of the 1-tree. Note that, by conveniently reordering the vertices of the 1tree, it is possible to express $K_{i}$ and $T_{i}$ as a circulant matrix and a triangular matrix, respectively, implying that $B_{i}$ is invertible. 
Therefore, a basis of (2), (3) is given by

$$
B=\left[\begin{array}{ll}
\bar{B} & 0 \\
\hat{B} & I
\end{array}\right],
$$

where $\bar{B}$ and $\hat{B}$ are submatrices of $\bar{A}$ and $\hat{A}$, respectively and

$$
\bar{B}=\left[\begin{array}{llll}
B_{1} & & & \\
& B_{2} & & \\
& & \ddots & \\
& & & B_{k}
\end{array}\right] .
$$

Because $\bar{B}$ is a block diagonal matrix whose blocks are nonsingular, $\bar{B}$ is nonsingular as well, implying that $B$ is a basis.

Theorems 3.1 and 3.2 establish a precise correspondence between bases of (LP) and special subgraphs of $G$. In the next theorem, this correspondence is extended to basic solutions of (LP).

Theorem 3.3. Let $B \in \mathcal{B}$ be a basis of $(L P)$ and let $\bar{x}$ be the basic solution associated to $B$. Then:

(i) all isolated vertices of $G_{B}$ index 0 -valued components of $\bar{x}$;

(ii) all vertices in tree components of $G_{B}$ index $(0,1)$-valued components of $\bar{x}$;

(iii) all vertices in 1-tree components of $G_{B}$ index $\frac{1}{2}$-valued components of $\bar{x}$.

Proof. The result immediately follows from (5), (6) and Lemma 3.1. Note that, given a tree component $C_{i}\left(V_{i}, E_{i}\right), i \in I_{0}$, the assignment of binary values to its vertices is uniquely determined by $v_{i}=V_{i} \backslash B$, the only nonbasic vertex of $C_{i}$, which takes value 0 .

For each $i \in I_{1}$ define $\kappa\left(C_{i}\right)$ as its unique cycle.

Theorem 3.4. [9] For every $B \in \mathcal{B}$, the corner polyedron of (STAB) associated to $B$ is 
$\operatorname{corner}(B)=\left\{(x, y) \in \mathbb{R}^{n+m}: A x+y=\mathbf{1}, x_{N} \geq 0, y_{N} \geq 0, \sum_{e \in \kappa\left(C_{i}\right)} y_{e} \geq 1, i \in I_{1}\right\}$

Let us denote by $\mathcal{B}_{+}=\left\{B \in \mathcal{B}: B^{-1} \mathbf{1} \geq \mathbf{0}\right\}$ the set of feasible bases and by $\operatorname{int}\left(\mathcal{B}_{+}\right)$the intersection of the corner polyhedra associated to all feasible bases, that is

$$
\operatorname{int}\left(\mathcal{B}_{+}\right)=\bigcap_{B \in \mathcal{B}_{+}} \operatorname{corner}(B)
$$

and define $\mathcal{C}$ as the set of all the induced odd cycles of $G(V, E)$.

Theorem 3.5. [9] $\operatorname{int}(\mathcal{B})=\operatorname{int}\left(\mathcal{B}_{+}\right)=\bar{S}(G)$, where

$$
\bar{S}(G)=\left\{(x, y) \in \mathbb{R}_{+}^{n+m}: A x+y=1, \sum_{e \in \mathcal{C}} y_{e} \geq 1, \forall C \in \mathcal{C}\right\} .
$$

Theorem 3.6. [9] For the stable set formulation (STAB), the set $\bar{S}(G)$, the split closure, the Chvátal closure, the $\left\{0, \frac{1}{2}\right\}$-Chvátal closure, int $(\mathcal{B})$ and $\operatorname{int}\left(\mathcal{B}_{+}\right)$are all identical.

\section{Properties of the edge formulation}

In this section we introduce some useful results about the edge relaxation polytope $L P(G)$, due mainly to Nemhauser and Trotter [11]. We also show that, in order to prove Theorems 2.1-2.4, it is sufficient to consider the case where the optimal solution to (LP) is $x_{i}^{*}=\frac{1}{2} \forall i=1, \ldots, n$.

Theorem 4.1. [10, 11, 12] Let $x$ be an extreme point of $L P(G)$. Then, $x_{i}=0, \frac{1}{2}$ or 1 for $i=1, \ldots, n$.

Theorem 4.2. [11] Suppose $x^{*}$ is an optimal $\left(0, \frac{1}{2}, 1\right)$-valued solution of (LP). Define sets $P=\left\{i \in V: x_{i}^{*}=1\right\}$ and $\bar{P}=\left\{i \in V: x_{i}^{*}=0\right\}$. There exists a maximum stable set in $G$ that contains $P$ and does not contain $\bar{P}$.

In other words, those variables which assume binary values in an optimal solution of (LP) retain the same values in some optimal solution of (STAB). 
This implies that to solve (STAB), one can solve (LP) and then find a stable set on the subgraph of $G$ induced by the vertices $i \in V: x_{i}^{*}=\frac{1}{2}$.

For any $P \subseteq V$ define the neighbors of $P$ as $N(P)=\{j \in V \backslash P:(i, j) \in$ $E$ for some $i \in P\}$.

The next theorem establishes a necessary and sufficient condition for $x_{i}^{*}=$ $\frac{1}{2} \forall i=1, \ldots, n$ to be a (unique) optimal solution of (LP).

Theorem 4.3. [11] The solution $x_{i}^{*}=\frac{1}{2} \forall i=1, \ldots, n$ is an optimal (resp. the unique optimal) solution of (LP) if and only if $|P| \leq|N(P)|$ (resp. $|P|<|N(P)|)$ for every nonempty stable set $P$.

In the next lemma we show that dropping nonnegativity constraints on all $x$ variables from (STAB) does not affect the optimal value when $x_{i}^{*}=$ $\frac{1}{2} \forall i=1, \ldots, n$ is an optimal solution of (LP).

Lemma 4.1. Given a graph $G(V, E)$, suppose that $x_{i}^{*}=\frac{1}{2}, i=1, \ldots, n$ is optimal for (LP). Define (NSTAB) as the problem obtained from (STAB) by dropping nonnegativity on the $x$ variables. Then:

(i) (NSTAB) has an optimal 0-1 solution;

(ii) if $x^{*}$ is the unique optimal solution to (LP), all optimal solutions to (NSTAB) are 0-1.

Proof. For simplicity, we write (NSTAB) as:

$$
\begin{array}{lll}
\max & \sum_{i=1}^{n} x_{i} & \\
\text { s.t. } & x_{i}+x_{j} \leq 1 & \forall(i, j) \in E \\
& x_{i} \in \mathbb{Z}^{n} & \forall i \in V .
\end{array}
$$

This avoids dealing with the $y$ variables.

First, note that (NSTAB) has a feasible solution (the 0 vector). Second, observe that (NSTAB) is bounded because $x^{*}$ is optimal for its LP relaxation (this follows from the fact that we remove from (LP) only constraints that are not tight at $x^{*}$, i.e. nonnegativity on the $x$ variables). Therefore (NSTAB) has an optimal solution.

$(i)$ : Assume that $x_{i}^{*}=1 / 2, i=1, \ldots, n$ is optimal for (LP) but not necessarily unique. For any feasible solution $\hat{x}$ to (NSTAB), define $S_{-}(\hat{x})$ : $\left\{i \in V: \hat{x}_{i}<0\right\}, S_{+}(\hat{x}):\left\{i \in V: \hat{x}_{i}>1\right\}$. Observe that every vertex in 
$S_{+}(\hat{x})$ can only be adjacent to vertices in $S_{-}(\hat{x})$. Therefore the incidence vector of $S_{+}(\hat{x})$ defines a stable set of $G$. By Theorem 4.3, this implies $\left|S_{-}(\hat{x})\right| \geq\left|S_{+}(\hat{x})\right|$.

Let $\bar{x}$ be an optimal solution to (NSTAB). If $S_{-}(\bar{x})=\emptyset$, we are done. Define $\Delta(\bar{x})=\min _{i \in V}\left\{\bar{x}_{i}\right\}$. Note that $\Delta(\bar{x}) \leq-1$. Construct a solution $\tilde{x}$ as:

$$
\tilde{x}_{k}=\left\{\begin{array}{lll}
\bar{x}_{k} & \text { for } \quad k \in V \backslash\left(S_{-}(\bar{x}) \cup S_{+}(\bar{x})\right) \\
\bar{x}_{k}-1 & \text { for } \quad k \in V \cap S_{+}(\bar{x}) \\
\bar{x}_{k}+1 & \text { for } \quad k \in V \cap S_{-}(\bar{x}) .
\end{array}\right.
$$

We show that $\tilde{x}$ satisfies all the edge constraints. It suffices to prove that increasing by 1 a variable $x_{i}$ with $i \in S_{-}(\bar{x})$ does not yield constraint violations. Observe that $\tilde{x}_{i} \leq 0$. Let $j$ be a vertex adjacent to $i$. Either:

- $j \in S_{+}(\bar{x})$ and $\tilde{x}_{j}=\bar{x}_{j}-1$, or

- $j \in S_{-}(\bar{x})$ and $\tilde{x}_{j} \leq 0$, or

- $j \in V \backslash\left(S_{-}(\bar{x}) \cup S_{+}(\bar{x})\right)$ and $\tilde{x}_{j}=\bar{x}_{j} \leq 1$.

In all cases, $\tilde{x}_{i}+\tilde{x}_{j} \leq 1$. Therefore $\tilde{x}$ is feasible for (NSTAB) and $\Delta(\tilde{x})=$ $\Delta(\bar{x})+1$. The objective value of $\tilde{x}$ is $\sum_{i \in V} \tilde{x}_{i}=\sum_{i \in V} \bar{x}_{i}+\left|S_{-}(\bar{x})\right|-\left|S_{+}(\bar{x})\right| \geq$ $\sum_{i \in V} \bar{x}_{i}$, so $\tilde{x}$ is optimal. We can iterate this construction from $\tilde{x}$ until we obtain an optimal solution $x^{\prime}$ with $\Delta\left(x^{\prime}\right)=0$, i.e., $S_{-}\left(x^{\prime}\right)=\emptyset$. This implies that $x^{\prime}$ has $0-1$ components.

(ii): Observe that if $x_{i}^{*}=1 / 2, i=1, \ldots, n$ is the unique optimum of (LP), by Theorem $4.3\left|S_{-}(\hat{x})\right|>\left|S_{+}(\hat{x})\right|$. Let $\bar{x}$ be an optimal solution to (NSTAB) and suppose $S_{-}(\bar{x}) \neq \emptyset$. Construct $\tilde{x}$ as shown above. $\tilde{x}$ has cost $\sum_{i \in V} \bar{x}_{i}+\left|S_{-}(\bar{x})\right|-\left|S_{+}(\bar{x})\right|>\sum_{i \in V} \bar{x}_{i}$. This contradicts optimality of $\bar{x}$, therefore $S_{-}(\bar{x})=\emptyset$, i.e. $\bar{x}$ is $0-1$.

We now show that, to prove Theorems 2.1-2.4, it is sufficient to restrict our attention to the case where the optimum of (LP) is $x_{i}^{*}=1 / 2, i=1, \ldots, n$.

Given an optimal solution $x^{*}$ to (LP), let $V^{0}=\left\{i \in V: x_{i}^{*}=0\right\}, V^{\frac{1}{2}}=$ $\left\{i \in V: x_{i}^{*}=\frac{1}{2}\right\}, V^{1}=\left\{i \in V: x_{i}^{*}=1\right\}$. Define $E^{00}=\{(i, j) \in E:$ $\left.i, j \in V^{0}\right\}, E^{0 \frac{1}{2}}=\left\{(i, j) \in E: i \in V^{0}, j \in V^{\frac{1}{2}}\right\}, E^{01}=\{(i, j) \in E: i \in$ $\left.V^{0}, j \in V^{1}\right\}, E^{\frac{1}{2} \frac{1}{2}}=\left\{(i, j) \in E: i, j \in V^{\frac{1}{2}}\right\}$ (the graph being undirected, the edges are unordered pairs). By Theorem 4.1, $V^{0}, V^{\frac{1}{2}}, V^{1}$ defines a partition of $V$. Since there can be no edge between $V^{1}$ and $V^{\frac{1}{2}} \cup V^{1}$, it follows that $E^{00}, E^{0 \frac{1}{2}}, E^{01}, E^{\frac{1}{2} \frac{1}{2}}$ is a partition of $E$. We consider two induced subgraphs 
of $G$ : $G^{\frac{1}{2}}$ induced by $V^{\frac{1}{2}}$, and $G^{01}$ induced by $V^{0} \cup V^{1}$. We show that for all relaxations of (STAB) studied in this paper, if we are able to compute a bound on $G^{\frac{1}{2}}$, we can generalize its value to $G$ by simply adding $\left|V^{1}\right|=p$.

Theorem 4.4. Let $x^{*}$ be the optimal solution to (LP) and let $B$ be an optimal basis associated to $x^{*}$. Partition $x^{*}$ according to $V^{\frac{1}{2}}$ and $V^{0} \cup V^{1}$ as $\left(x^{* \frac{1}{2}}, x^{* 01}\right)$. Define $B^{01}=B \cap\left(V^{0} \cup V^{1} \cup E^{00} \cup E^{01}\right)$ and $B^{\frac{1}{2}}=B \cap\left(V^{\frac{1}{2}} \cup E^{\frac{1}{2} \frac{1}{2}}\right)$. Let $G^{01}=G\left[V^{0} \cup V^{1}\right]$ and $G^{\frac{1}{2}}=G\left[V^{\frac{1}{2}}\right]$. Then:

(i) if $\tilde{B}^{\frac{1}{2}}$ is an optimal basis associated to $x^{* \frac{1}{2}}$ for $\max \left\{\mathbf{1}^{T} x: x \in L P\left(G^{\frac{1}{2}}\right)\right\}$ and $\tilde{B}=B^{01} \cup \tilde{B}^{\frac{1}{2}} \cup E^{0 \frac{1}{2}}$, then:

1. $\tilde{B}$ is a basis of $(L P)$,

2. $\max \left\{\mathbf{1}^{T} x: x \in \operatorname{corner}(\tilde{B})\right\}=p+\max \left\{\mathbf{1}^{T} x: x \in \operatorname{corner}\left(\tilde{B}^{\frac{1}{2}}\right)\right\}$,

3. $\max \left\{\mathbf{1}^{T} x: x \in L P(G) \cap \operatorname{corner}(\tilde{B})\right\}=p+\max \left\{\mathbf{1}^{T} x: x \in L P\left(G^{\frac{1}{2}}\right) \cap\right.$ $\left.\operatorname{corner}\left(\tilde{B}^{\frac{1}{2}}\right)\right\}$;

(ii) if $\mathcal{B}^{\frac{1}{2}}$ is the set of all bases of $\max \left\{\mathbf{1}^{T} x: x \in L P\left(G^{\frac{1}{2}}\right)\right\}$, then: $\max \left\{\mathbf{1}^{T} x: x \in\right.$ $\operatorname{int}(\mathcal{B})\}=p+\max \left\{\mathbf{1}^{T} x: x \in \operatorname{int}\left(\mathcal{B}^{\frac{1}{2}}\right)\right\} ;$

(iii) $\max \left\{\mathbf{1}^{T} x: x \in \operatorname{strict}\left(x^{*}\right)\right\}=p+\max \left\{\mathbf{1}^{T} x: x \in \operatorname{strict}\left(x^{* \frac{1}{2}}\right)\right\}$.

Proof. First, observe that the constraints corresponding to edges in $E^{0 \frac{1}{2}}$ are not tight at $x^{*}$. Therefore they can be relaxed without affecting optimality of $x^{*}$ for (LP). This implies that $\max \left\{\mathbf{1}^{T} x: x \in L P(G)\right\}=\max \left\{\mathbf{1}^{T} x: x \in\right.$ $\left.L P\left(G^{01}\right)\right\}+\max \left\{\mathbf{1}^{T} x: x \in L P\left(G^{\frac{1}{2}}\right)\right\}$ and $x^{* 01}$ is optimal on $L P\left(G^{01}\right)$. Since $x^{* 01}$ is in $S\left(G^{01}\right)$, it is an optimal stable set in $G^{01}$ and $\max \left\{\mathbf{1}^{T} x: x \in\right.$ $\left.S\left(G^{01}\right)\right\}=\max \left\{\mathbf{1}^{T} x: x \in L P\left(G^{01}\right)\right\}$.

Let $\mathcal{B}^{01}$ be the set of all bases of $\max \left\{\mathbf{1}^{T} x: x \in L P\left(G^{01}\right)\right\}$. We have the chains:

$$
\begin{aligned}
\max \left\{\mathbf{1}^{T} x: x \in S\left(G^{01}\right)\right\} & \leq \max \left\{\mathbf{1}^{T} x: x \in \operatorname{strict}\left(x^{* 01}\right)\right\} \leq \\
\max \left\{\mathbf{1}^{T} x: x \in \operatorname{corner}\left(B^{01}\right)\right\} & \leq \max \left\{\mathbf{1}^{T} x: x \in L P\left(G^{01}\right)\right\}= \\
\max \left\{\mathbf{1}^{T} x: x \in S\left(G^{01}\right)\right\} &
\end{aligned}
$$

and

$$
\begin{aligned}
\max \left\{\mathbf{1}^{T} x: x \in S\left(G^{01}\right)\right\} & \leq \max \left\{\mathbf{1}^{T} x: x \in \operatorname{int}\left(\mathcal{B}^{01}\right)\right\} \leq \\
\max \left\{\mathbf{1}^{T} x: x \in\right. & \left.\leq P\left(G^{01}\right) \cap \operatorname{corner}\left(B^{01}\right)\right\} \\
\max \left\{\mathbf{1}^{T} x: x \in S\left(G^{01}\right)\right\}, & \leq \max \left\{\mathbf{1}^{T} x: x \in L P\left(G^{1}\right)\right\}=
\end{aligned}
$$


which imply that $x^{* 01}$ is optimal for all the relaxations discussed above on $G^{01}$, with cost $\left|V^{1}\right|=p$.

(i). $\tilde{B}$ has $m$ elements, and the subgraph $G_{\tilde{B}}$ corresponds to the union of the tree components of $G_{B}$ and the 1-tree components of $G_{\tilde{B}^{\frac{1}{2}}}$. Therefore, by Theorems 3.2 and $3.3, \tilde{B}$ is a basis of $L P(G)$, which proves $(i)-1$.

Observe that the $y$ variables corresponding to the constraints $E^{0 \frac{1}{2}}$ are basic in $\tilde{B}$. Therefore, they become free variables in corner $(\tilde{B})$ and the constraints $E^{0 \frac{1}{2}}$ can be dropped. Since there are no constraints linking $G^{01}$ and $G^{\frac{1}{2}}$ in corner $(\tilde{B})$, we have that $\max \left\{\mathbf{1}^{T} x: x \in \operatorname{corner}(\tilde{B})\right\}=\max \left\{\mathbf{1}^{T} x: x \in\right.$ corner $\left.\left(B^{01}\right)\right\}+\max \left\{\mathbf{1}^{T} x: x \in \operatorname{corner}\left(\tilde{B}^{\frac{1}{2}}\right)\right\}=p+\max \left\{\mathbf{1}^{T} x: x \in \operatorname{corner}\left(\tilde{B}^{\frac{1}{2}}\right)\right\}$. This proves $(i)-2$.

For $(i)-3$, we note that $\max \left\{\mathbf{1}^{T} x: x \in L P(G) \cap \operatorname{corner}(\tilde{B})\right\} \leq \max \left\{\mathbf{1}^{T} x: x \in\right.$ $\left.L P\left(G^{01}\right) \cap \operatorname{corner}\left(B^{01}\right)\right\}+\max \left\{\mathbf{1}^{T} x: x \in L P\left(G^{\frac{1}{2}}\right) \cap \operatorname{corner}\left(\tilde{B}^{\frac{1}{2}}\right)\right\}$ because by optimizing separately over $L P\left(G^{01}\right) \cap \operatorname{corner}\left(B^{01}\right)$ and $L P\left(G^{\frac{1}{2}}\right) \cap \operatorname{corner}\left(\tilde{B}^{\frac{1}{2}}\right)$ we are relaxing the edge constraints $E^{0 \frac{1}{2}}$ that are present in $\operatorname{LP} \cap \operatorname{corner}(\tilde{B})$. Observe that any optimal solution to $\max \left\{\mathbf{1}^{T} x: x \in L P\left(G^{\frac{1}{2}}\right) \cap \operatorname{corner}\left(\tilde{B}^{\frac{1}{2}}\right)\right\}$ has components in $[0,1]$. Pick any such solution $\tilde{x}^{\frac{1}{2}}$. Define $\hat{x}$ as:

$$
\hat{x}_{i}=\left\{\begin{array}{cll}
\tilde{x}_{i}^{\frac{1}{2}} & \text { for } \quad i \in V^{\frac{1}{2}} \\
x_{i}^{*} & \text { for } \quad i \in V^{0} \cup V^{1} .
\end{array}\right.
$$

Clearly $\hat{x}$ satisfies the constraints of $L P\left(G^{01}\right) \cap \operatorname{corner}\left(B^{01}\right)$ and $L P\left(G^{\frac{1}{2}}\right) \cap$ $\operatorname{corner}\left(\tilde{B}^{\frac{1}{2}}\right)$. Additionally, it satisfies the edge constraints $E^{0 \frac{1}{2}}$ because the variables corresponding to vertices in $V^{0}$ have value 0 and those in $V^{\frac{1}{2}}$ have value in $[0,1]$. Thus, $\hat{x}$ is feasible for $L P(G) \cap \operatorname{corner}(\tilde{B})$ with cost $\max \left\{\mathbf{1}^{T} x: x \in L P\left(G^{01}\right) \cap \operatorname{corner}\left(B^{01}\right)\right\}+\max \left\{\mathbf{1}^{T} x: x \in L P\left(G^{\frac{1}{2}}\right) \cap \operatorname{corner}\left(\tilde{B}^{\frac{1}{2}}\right)\right\}=$ $p+\max \left\{\mathbf{1}^{T} x: x \in L P\left(G^{\frac{1}{2}}\right) \cap \operatorname{corner}\left(\tilde{B}^{\frac{1}{2}}\right)\right\}$, and therefore optimal.

(ii). Recall the description of $\operatorname{int}(\mathcal{B})$ given in Theorem 3.5. Observe that $\max \left\{\mathbf{1}^{T} x: x \in \operatorname{int}(\mathcal{B})\right\} \leq \max \left\{\mathbf{1}^{T} x: x \in \operatorname{int}\left(\mathcal{B}^{01}\right)\right\}+\max \left\{\mathbf{1}^{T} x: x \in \operatorname{int}\left(\mathcal{B}^{\frac{1}{2}}\right)\right\}$ because by optimizing separately over $\operatorname{int}\left(\mathcal{B}^{01}\right)$ and $\operatorname{int}\left(\mathcal{B}^{\frac{1}{2}}\right)$ we are relaxing some of the constraints that $\operatorname{define} \operatorname{int}(\mathcal{B})$, namely: the edge constraints $E^{0 \frac{1}{2}}$, and the odd cycle inequalities involving at least one edge in $E^{0 \frac{1}{2}}$. Let $\tilde{x}^{\frac{1}{2}}$ be an optimal solution to $\max \left\{\mathbf{1}^{T} x: x \in \operatorname{int}\left(\mathcal{B}^{\frac{1}{2}}\right)\right\}$. Define $\hat{x}$ as in (10). By construction, $\hat{x}$ satisfies the constraints of $\operatorname{int}\left(\mathcal{B}^{01}\right)$ and $\operatorname{int}\left(\mathcal{B}^{\frac{1}{2}}\right)$. Since $\hat{x}_{i}=$ $0 \forall i \in V_{0}$, it also satisfies all the edge constraints $E^{0 \frac{1}{2}}\left(\tilde{x}^{\frac{1}{2}}\right.$ has components in $[0,1]$ ) and any odd cycle inequality involving at least one edge in $E^{0 \frac{1}{2}}$ (for 
any such cycle $\kappa$ with $2 k+1$ edges, no more than $2 k$ vertices are in $V^{\frac{1}{2}}$; since they form a chain, the vertices in the cycle add up to at most $k$ ). Thus, $\hat{x}$ is feasible for $\operatorname{int}(\mathcal{B})$ with cost $\max \left\{\mathbf{1}^{T} x: x \in \operatorname{int}\left(\mathcal{B}^{01}\right)\right\}+\max \left\{\mathbf{1}^{T} x: x \in\right.$ $\left.\operatorname{int}\left(\mathcal{B}^{\frac{1}{2}}\right)\right\}=p+\max \left\{\mathbf{1}^{T} x: x \in \operatorname{int}\left(\mathcal{B}^{\frac{1}{2}}\right)\right\}$, and therefore optimal.

(iii). We have $\max \left\{\mathbf{1}^{T} x: x \in \operatorname{strict}\left(x^{*}\right)\right\} \leq \max \left\{\mathbf{1}^{T} x: x \in \operatorname{strict}\left(x^{* 01}\right)\right\}+$ $\max \left\{\mathbf{1}^{T} x: x \in \operatorname{strict}\left(x^{* \frac{1}{2}}\right)\right\}$ since the edge constraints $E^{0 \frac{1}{2}}$ are relaxed when optimizing separately over $\operatorname{strict}\left(x^{* 01}\right)$ and $\operatorname{strict}\left(x^{* \frac{1}{2}}\right)$. By Lemma 4.1 , there exists an optimal $0-1$ solution to $\max \left\{\mathbf{1}^{T} x: x \in \operatorname{strict}\left(x^{* \frac{1}{2}}\right)\right\}$. Let $\tilde{x}^{\frac{1}{2}}$ be such a solution, and define $\hat{x}$ as in (10). Observe that $\hat{x}$ is $0-1$ and satisfies all the edge constraints, including those in $E^{0 \frac{1}{2}}$ because $x_{i}=0 \forall i \in V^{0}$. This implies that $\hat{x}$ is feasible for $\operatorname{strict}\left(x^{*}\right)$ with cost $\max \left\{\mathbf{1}^{T} x: x \in \operatorname{strict}\left(x^{* 01}\right)\right\}+$ $\max \left\{\mathbf{1}^{T} x: x \in \operatorname{strict}\left(x^{* \frac{1}{2}}\right)\right\}=p+\max \left\{\mathbf{1}^{T} x: x \in \operatorname{strict}\left(x^{* \frac{1}{2}}\right)\right\}$, and therefore optimal.

By Theorem 4.4, the bound provided by a relaxation on $G^{\frac{1}{2}}$ is sufficient to characterize the bound by the same kind of relaxation on $G$. In particular, for the corner relaxation and LP^corner we can take any basis of the LP on $G^{\frac{1}{2}}$, and there always exists a basis of the LP on the full graph $G$ for which the generalization of the bound on $G^{\frac{1}{2}}$ is valid.

\section{Optimizing over the corner relaxation}

We assume that $x_{i}^{*}=\frac{1}{2} \forall i=1, \ldots, n$ is an optimal solution to (LP). Thus $z_{\mathrm{LP}}=\frac{n}{2}$. If $m>n$, there are many bases associated to vertex $x^{*}$, which may yield different corner relaxations. We show that the strength of these relaxations can be significantly different. We prove that if the graph is connected and its maximum matching has size $\left\lfloor\frac{n}{2}\right\rfloor$, there exists an optimal basis associated to $x^{*}$ yielding a bound of $\left\lfloor\frac{n}{2}\right\rfloor$, i.e. a weak bound improvement over (LP). On the other hand, if the graph can be partitioned into triangles, we show that there is also a basis providing the much stronger bound of $\frac{n}{3}$. In the classical random graph model where edges occur independently with a fixed probability $p$, both of the above conditions hold almost surely (i.e. with probability going to 1 as the number of vertices $n$ increases) [14] when $n$ is a multiple of 3 . This implies that almost all graphs have both a weak corner relaxation with bound $\left\lfloor\frac{n}{2}\right\rfloor$ and a much stronger one with bound of the order of $\frac{n}{3}$.

For each basis $B \in \mathcal{B}$ associated to $x^{*}$, all $x$ variables are positive and belong to $B$. In the corner polyhedron we drop the nonnegativity constraints 
on variables $y_{i j}$ such that $(i, j) \in E_{B}$. This corresponds to removing the redundant constraints of type $x_{i}+x_{j}+y_{i j}=1$ for each $(i, j) \in E_{B}$. Thus, the corner polyhedron associated to $B$ is the convex hull of the points satisfying

$$
\begin{array}{ll}
x_{i}+x_{j}+y_{i j}=1 & \forall(i, j) \in E_{N} \\
y_{i j} \geq 0 & \forall(i, j) \in E_{N} \\
x_{i} \in \mathbb{Z}^{n} & \forall i \in V .
\end{array}
$$

Using the structure described in Section 3, we show that any basis $B$ associated to $x^{*}$ has, in general, an associated graph $G_{B}$ with $k \geq 1$ connected components, each one representing a 1-tree.

Lemma 5.1. Any basis $B$ associated to vertex $x_{i}^{*}=\frac{1}{2} \forall i=1, \ldots, n$ is such that all connected components $C_{1}, \ldots, C_{k}, k \geq 1$ are 1-trees.

Proof. By contradiction, suppose this is not the case, that is, there exists at least one connected component $C_{i}\left(V_{i}, E_{i}\right)$ which is a tree. Then, by Theorem 3.3, some components of $x^{*}$ would have binary values, precisely $x_{j}^{*} \in\{0,1\} \forall j \in V_{i}$.

Define

$$
P(B)=\left\{(x, y): A x+y=1, x_{N} \geq 0, y_{N} \geq 0\right\} .
$$

$P(B)$ is a pointed cone defined by the sum of the LP optimum $x^{*}$ and a conic combination of the nonbasic rays. Note that the cone $P(B)$ is precisely the linear relaxation arising from (11). Given any (MILP), consider the problem obtained from the linear relaxation by dropping nonnegativity on basic variables. If this latter problem contains a point satisfying the integrality constraints of (MILP), any basis $B$ of the linear relaxation is optimal if and only if corner $(B)$ has an optimal solution. For simplicity, we prove this result for the edge formulation of the stable set problem, but Lemma 5.2 applies to any MILP.

Lemma 5.2. Suppose $x_{i}^{*}=\frac{1}{2} \forall i=1, \ldots, n$ is an optimal solution of (LP). Any basis $B$ associated to $x^{*}$ is optimal for $(L P)$ if and only if corner $(B)$ has an optimal solution.

Proof. If $B$ is an optimal basis of (LP) associated to $x^{*}$, all the reduced costs of nonbasic variables are non-positive. The objective function can be rewritten in terms of the nonbasic variables as $\frac{n}{2}+\max \sum_{(i, j) \in E_{N}} c_{i j}^{\prime} y_{i j}$, where for all $(i, j) \in E_{N}, c_{i j}^{\prime}$ is the reduced cost of nonbasic variable $y_{i j}$. Because 
$c_{i j}^{\prime} \leq 0 \quad \forall(i, j) \in E \cap N$ and $y_{i j} \geq 0 \quad \forall(i, j) \in E_{N}$, optimizing over (11) is not unbounded. As (11) is non-empty, $\operatorname{corner}(B)$ has an optimal solution. Assume now that problem $(\operatorname{corner}(B))$ has an optimal solution and, by contradiction, suppose $c_{u v}^{\prime}>0$ for some $(u, v) \in E_{N}$. It is possible to increase variable $y_{u v}$ by a positive integer $M$, without modifying any of the other (nonbasic) $y$ variables, in such a way that all $x_{i}$ are integer (because all $x_{i}$ 's are basic and unrestricted in sign). This would yield an improvement of the objective function equal to $M c_{u v}^{\prime}$, showing that $(\operatorname{corner}(B))$ is unbounded for $M \rightarrow \infty$.

Definition 5.1. A bipartite graph $B(U, V, W)$ is balanced if $|U|=|V|$.

Definition 5.2. A bipartite graph $B(U, V, W)$ is nearly balanced if $|U|-$ $|V|= \pm 1$.

Definition 5.3. We define a 1-tree component $C_{i}\left(V_{i}, E_{i}\right)$ to be unbalanced if it admits a stable set $P_{i} \subseteq V_{i}$ such that $2\left|P_{i}\right| \geq\left|V_{i}\right|+1$. Otherwise, we say that it is balanced.

Lemma 5.3. Let $B$ be a basis of $(L P)$ associated to $x^{*}$, and let $G_{B}$ be the corresponding graph as defined in Section 3. The basis $B$ is optimal if and only if $x^{*}$ is an optimal solution of $(L P)$ over $G_{B}$.

Proof. Suppose $B$ is an optimal basis of (LP). Relaxing nonnegativity constraints of basic variables of $L P(G)$ corresponds to optimizing on $L P\left(G_{B}\right)$. Because $B$ is an optimal basis, dropping the constraints associated to basic variables does not affect optimality of $x^{*}$. For the converse, suppose $x^{*}$ is an optimal solution on $L P\left(G_{B}\right)$. By Lemma 4.1, (NSTAB) admits a 0-1 optimal solution on $G_{B}$. Remark that the latter problem is exactly corner $(B)$. This implies that corner $(B)$ has an optimal solution and therefore, by Lemma 5.2, $B$ is an optimal basis of (LP).

Lemma 5.4. Suppose $B$ is an optimal basis of $(L P)$ associated to $x^{*}$. Then all its components $C_{i}$ are balanced.

Proof. By Lemma 5.3, optimizing over $L P\left(G_{B}\right)$ gives an upper bound of $\frac{n}{2}$ for (STAB) over $G_{B}$. This implies that the maximum stable set of $G_{B}$ has size at most $\left\lfloor\frac{n}{2}\right\rfloor$.

Lemma 5.5. If $C(V, E)$ is a balanced 1-tree containing an odd cycle, then there exists an edge $\left(u_{j}, u_{j+1}\right) \in \kappa(C)$ such that $C^{\prime}\left(V, E \backslash\left(u_{j}, u_{j+1}\right)\right)$ is a bipartite graph which is nearly balanced if $|V|$ is odd, or balanced if $|V|$ is even. 
Proof. Suppose that $|\kappa(C)|=k$ and let $u_{1}, u_{2}, \ldots, u_{k}, u_{k+1}=u_{1}$ be the vertices of $\kappa(C)$. By contradiction, suppose that for all $j=1, \ldots, k$ the removal of edge $\left(u_{j}, u_{j+1}\right)$ yields the tree $T_{j}\left(V_{j}^{+}, V_{j}^{-}, E \backslash\left(u_{j}, u_{j+1}\right)\right)$, such that $\left|V_{j}^{+}\right| \geq\left|V_{j}^{-}\right|+2$. As there is a path of even length connecting $u_{j}$ and $u_{j+1}$, they both belong to the same side of the partition. They cannot belong both to $V_{j}^{-}$, otherwise $V_{j}^{+}$would be a stable set of $C$ such that $2\left|V_{j}^{+}\right| \geq\left|V_{j}^{+}\right|+\left|V_{j}^{-}\right|+2=|V|+2$ and therefore $C$ would be unbalanced. Thus, $u_{j}, u_{j+1} \in V_{j}^{+}$.

We first prove that the inequality $\left|V_{j}^{+}\right| \geq\left|V_{j}^{-}\right|+2$ cannot hold strictly. Suppose otherwise. The stable set $P=V_{j}^{+} \backslash\left\{u_{j}\right\}$ would be such that $2|P| \geq$ $|V|+1$, implying again that $C$ is unbalanced. Thus, it can only be $\left|V_{j}^{+}\right|=$ $\left|V_{j}^{-}\right|+2$. If $|V|$ is odd, this is not possible and the first part of the statement is proven.

Consider therefore the case where $|V|$ is even. Observe that $C$ can be partitioned into $k$ branches $B\left(u_{j}\right)$, one departing from each vertex of the odd cycle $u_{j}, j=1, \ldots, k$. By contradiction, suppose that for all $j=1, \ldots, k$ removing $\left(u_{j}, u_{j+1}\right)$ yields a tree $T_{j}$ whose bipartition satisfies $\left|V_{j}^{+}\right|=\left|V_{j}^{-}\right|+2$. Remark that, when we remove two consecutive edges $\left(u_{j-1}, u_{j}\right)$ and $\left(u_{j}, u_{j+1}\right)$ the corresponding trees $T_{j-1}$ and $T_{j}$ are such that: $V_{j-1}^{+} \cap B\left(u_{j}\right)=V_{j}^{+} \cap$ $B\left(u_{j}\right)$ and $V_{j-1}^{-} \cap B\left(u_{j}\right)=V_{j}^{-} \cap B\left(u_{j}\right) ; V_{j-1}^{+} \backslash B\left(u_{j}\right)=V_{j}^{-} \backslash B\left(u_{j}\right)$ and $V_{j-1}^{-} \backslash B\left(u_{j}\right)=V_{j}^{+} \backslash B\left(u_{j}\right)$. Following this remark and recalling that $2=$ $\left|V_{j}^{+}\right|-\left|V_{j}^{-}\right|=\left|V_{j-1}^{+}\right|-\left|V_{j-1}^{-}\right|$, by elementary algebraic manipulations it follows that $\left|B\left(u_{j}\right) \cap V_{j}^{+}\right|=\left|B\left(u_{j}\right) \cap V_{j}^{-}\right|+2$. Therefore, one can build a stable set $P$ of $C$ by selecting in each branch $B\left(u_{j}\right)$, all vertices in $B\left(u_{j}\right) \cap V_{j}^{+}$, and by including or excluding $u_{j}$ depending on $j$ being even or odd, i.e.

$$
P=\left(\bigcup_{j=1, \ldots, k} V_{j}^{+}\right) \backslash\left(\bigcup_{\substack{j=1, \ldots, k \\ j \text { odd }}} u_{j}\right) .
$$

The corresponding stable set has therefore size $\frac{|V|+k-1}{2} \geq \frac{|V|}{2}+1$, contradicting the hypothesis that $C$ is a balanced 1-tree.

Lemma 5.6. Let $B \in \mathcal{B}$ be an optimal basis associated to $x^{*}$ and consider any 1-tree component $C_{i}\left(V_{i}, E_{i}\right)$ of $G_{B}$. There exists an edge $\left(u_{i}, v_{i}\right) \in \kappa\left(C_{i}\right)$ which, if removed, yields a tree with all of its stable sets $P_{i}$ satisfying $\left|P_{i}\right| \leq$ $\left\lceil\frac{\left|V_{i}\right|}{2}\right\rceil$. 
Proof. By Lemma 5.4, $C_{i}$ is balanced. By Lemma 5.5, we can remove an edge $\left(u_{i}, v_{i}\right) \in \kappa\left(C_{i}\right)$ in order to obtain a bipartite graph, which is balanced or nearly balanced depending on the parity of $\left|V_{i}\right|$. Let us denote by $V_{i}^{+}$and $V_{i}^{-}$the two sets of the bipartition and suppose w.l.o.g. $\left|V_{i}^{+}\right|=\left\lceil\frac{\left|V_{i}\right|}{2}\right\rceil$ and $\left|V_{i}^{-}\right|=\left\lfloor\frac{\left|V_{i}\right|}{2}\right\rfloor$.

By contradiction, suppose that there exists a stable set $P_{i}$ of $C_{i}^{\prime}\left(V_{i}, E_{i} \backslash\right.$ $\left.\left(u_{i}, v_{i}\right)\right)$ such that $\left|P_{i}\right|>\left\lceil\frac{\left|V_{i}\right|}{2}\right\rceil$. Consider $P_{i} \cap V_{i}^{+}$and $P_{i} \cap V_{i}^{-}$. Clearly neither $V_{i}^{+}$, nor $P_{i}$ are stable sets of $C_{i}$, because $C_{i}$ is a balanced 1-tree. This implies that $u_{i}, v_{i} \in P_{i} \cap V_{i}^{+}$. Because $B$ is optimal, by Lemma $5.3, x^{*}$ is optimal for the linear relaxation of (STAB) on $G_{B}$. A feasible solution $\tilde{x}$ for $L P\left(G_{B}\right)$ can be obtained as

$$
\tilde{x}_{j}=\left\{\begin{array}{lll}
0 & \text { for } & j \in V_{i}^{+} \backslash P_{i} \\
1 & \text { for } & j \in P_{i} \cap V_{i}^{-} \\
\frac{1}{2} & \text { for } & j \in\left(V_{i}^{-} \backslash P_{i}\right) \cup\left(P_{i} \cap V_{i}^{+}\right)
\end{array}\right.
$$

Because $\left|V_{i}^{-} \cap P_{i}\right|>\left|V_{i}^{+} \backslash P_{i}\right|$, for this latter solution $\sum_{i=1}^{n} \tilde{x}_{i} \geq \frac{\left|V_{i}\right|+1}{2}$ which contradicts optimality of $x^{*}$ on $L P\left(G_{B}\right)$.

Theorem 5.1. Consider (STAB) and its linear relaxation (LP). Suppose $x_{i}^{*}=\frac{1}{2} \forall i=1, \ldots, n$ is an optimal solution of $(L P)$ and $B$ is an optimal basis associated to $x^{*}$, composed by $k 1$-tree components $C_{i}\left(V_{i}, E_{i}\right), i=1, \ldots, k$. Then, $z_{\text {corner }(B)}=\frac{n-k_{o}}{2}$, where $k_{o} \leq k$ is the number of odd components among $C_{i}, i=1, \ldots, k$.

Proof. For all $i=1, \ldots, k$ define

$$
W_{i}=\left\{(x, y) \in \mathbb{Z}^{\left|V_{i}\right|} \times \mathbb{R}_{+}^{\left|E_{i}\right|}: x_{u}+x_{v}+y_{u v}=1 \quad \forall(u, v) \in E_{i}\right\}
$$

and partition $(x, y)$ according to components $C_{i}$ into $\left\{\left(x^{i}, y^{i}\right)\right\}, i=1, \ldots, k$. Problem $(\operatorname{corner}(B))$ can be split into $k$ independent problems, one for each component of the basis, because $z_{\operatorname{corner}(B)}=\sum_{i=1}^{k} z\left(C_{i}\right)$, where

$$
z\left(C_{i}\right)=\max \left\{\mathbf{1}^{T} x^{i}:\left(x^{i}, y^{i}\right) \in W_{i}\right\}
$$

By optimality of $B$ and Lemma 4.1, (13) admits an optimal $(0,1)$-valued solution $\left(x^{i}, y^{i}\right)$, such that $x^{i}$ is the incidence vector of a stable set $P_{i}$ of vertices in $C_{i}$. By Lemma 5.4 , because $B$ is an optimal basis, $C_{i}$ is balanced 
and therefore $\left|P_{i}\right|=z\left(C_{i}\right) \leq\left\lfloor\frac{\left|V_{i}\right|}{2}\right\rfloor$. By Lemma 5.5, there exists an edge $\left(u_{i}, v_{i}\right) \in \kappa\left(C_{i}\right)$ such that $C_{i}^{\prime}\left(V_{i}, E_{i} \backslash\left(u_{i}, v_{i}\right)\right)$ is a bipartite graph which is balanced or nearly balanced, depending on $\left|V_{i}\right|$ being even or odd, respectively. Moreover, by Lemma 5.6, $C_{i}^{\prime}$ is such that all its stable sets have size at most $\left[\frac{\left|V_{i}\right|}{2}\right]$. This implies that a maximum stable set of $C_{i}$ corresponds to the side of the bipartition of $C_{i}^{\prime}$ that has cardinality $\left\lfloor\frac{\left|V_{i}\right|}{2}\right\rfloor$ and does not contain $\left(u_{i}, v_{i}\right)$. Therefore, there always exists in $C_{i}$ a stable set of cardinality $\left\lfloor\frac{\left|V_{i}\right|}{2}\right\rfloor$, implying $z\left(C_{i}\right)=\left\lfloor\frac{\left|V_{i}\right|}{2}\right\rfloor$, which completes the proof of the theorem.

Next, we show that if the graph is connected and its maximum matching has size $\left\lfloor\frac{n}{2}\right\rfloor$, there always exists an optimal basis that has only one connected component.

Lemma 5.7. Let $G$ be a connected graph on $n$ vertices. Consider (STAB) and its linear relaxation (LP). Suppose $x_{i}^{*}=\frac{1}{2} \forall i=1, \ldots, n$ is an optimal solution of $(L P)$. There exists an optimal basis $B$ associated to $x^{*}$ which has only one connected component if and only if $G$ admits a perfect matching, if $n$ is even, or a nearly perfect matching, if $n$ is odd.

Proof. Let us first show that the condition is sufficient.

If $n$ is even and $G$ admits a perfect matching, it is possible to incrementally build a spanning tree of $G$, such that all edges of the perfect matching belong to the tree. This can be done by adding to the tree, at each iteration, first an edge of the matching, and then an edge of the cutset separating the vertices in the tree from the vertices outside the tree. By construction, a maximum stable set in this spanning tree has size $\frac{n}{2}$. Moreover, because the tree is bipartite, both sides of the bipartition correspond to maximum stable sets of the spanning tree. Now, recall that $G$ admits $x^{*}$ as an optimal solution of (LP). This implies that $G$ cannot be bipartite, hence there exists an edge between two vertices on the same side of the bipartition. Adding this edge to the spanning tree yields a 1-tree with an odd cycle. It follows that an optimal solution of (LP) on the 1-tree is $x^{*}$, proving that the 1-tree corresponds to an optimal basis.

If $n$ is odd and $G$ admits a nearly perfect matching, consider $G-v$, the graph obtained from $G$ by removing vertex $v$ and all its incident edges, where 
$v$ is the only exposed vertex of the matching. Remark that $G-v$ may not be connected. Because $G-v$ admits a perfect matching, every connected component has an even number of vertices. Applying the same procedure described for the case of $n$ even, it is possible to build a forest spanning $G-v$, in such a way that all the edges of the nearly perfect matching belong to the forest. The maximum stable set in this forest has cardinality $\frac{n-1}{2}$. It is then possible to connect $v$ to the forest, in order to obtain a spanning tree of $G$, whose maximum stable set has at most cardinality $\frac{n+1}{2}$. In this case, a maximum stable set of the tree is given by the side of its bipartition which contains $v$. Recalling that $x^{*}$ is an optimal solution of (LP), it follows that there cannot exist a stable set of size $\frac{n+1}{2}$. This implies that there exists an edge between two vertices in the side of the bipartition that contains $v$. We can add this edge to the tree, in order to obtain a 1-tree with an odd cycle, such that the optimal solution of (LP) on the 1-tree has value $\frac{n}{2}$.

In order to show the converse, recall that for a bipartite graph $B$ of $n$ vertices, $n=\nu(B)+\alpha(B)$, where $\nu(B)$ and $\alpha(B)$ are the size of the maximum matching and of the maximum stable set in $B$, respectively. By Lemma 5.6, given an optimal basis associated to $x^{*}$, which consists of a unique 1 -tree, it is possible to remove an edge of the odd cycle of the 1-tree, in order to obtain a tree such that its maximum stable set has cardinality $\left\lceil\frac{n}{2}\right\rceil$. This implies that in the same tree, the maximum matching has cardinality $\left\lfloor\frac{n}{2}\right\rfloor$. Therefore, $G$ has a perfect matching, if $n$ is even, or a nearly perfect matching, if $n$ is odd.

Theorem 5.2. Let $G$ be a connected graph on $n$ vertices. Consider (STAB) and its linear relaxation (LP). Suppose $x_{i}^{*}=\frac{1}{2} \forall i=1, \ldots, n$ is an optimal solution of $(L P)$. There exists an optimal basis $B$ associated to $x^{*}$ such that $z_{\text {corner }(B)}=\left\lfloor\frac{n}{2}\right\rfloor$ if and only if $G$ admits a perfect matching, if $n$ is even, or a nearly perfect matching, if $n$ is odd.

Proof. By Lemma 5.7 there exists an optimal basis associated to $x^{*}$, which is composed by a unique 1-tree if and only if $G$ admits a perfect matching, if $n$ is even, or a nearly perfect matching, if $n$ is odd. Under this assumption, applying Theorem 5.1 with $k=1$, it follows that $z_{\text {corner }(B)}=\frac{n-1}{2}$, if $n$ is odd, and $z_{\operatorname{corner}(B)}=\frac{n}{2}$ if $n$ is even.

Theorems 4.4 and 5.2 imply Theorem 2.1.

Theorem 5.2 highlights the unlucky possibility where a basis yields an extremely weak corner relaxation. On the other hand, there may be the 
chance of choosing a basis which provides a much stronger corner relaxation, as shown in the next theorem.

Theorem 5.3. Suppose that there exists a partition of $V$ into triangles, i.e., cliques of size 3. Then there is an optimal basis $B$ associated to $x^{*}$ such that $z_{\text {corner }(B)}=\frac{n}{3}$.

Proof. Such a basis has $\frac{n}{3}$ connected 1-tree components corresponding to the partition into triangles. By Theorem 5.1, because $k_{o}=\frac{n}{3}, z_{\operatorname{corner}(B)}=\frac{n}{3}$.

A sufficient condition for $V$ to be partitioned into triangles is established by [15] and amounts to requiring that the minimum vertex degree is at least $\frac{2}{3} n$. A random graph $G(n, p)$ almost surely has such a partition whenever $n=3 k$ and $p \geq O\left(\frac{1}{n^{0.6}}\right)[16]$.

\section{Optimizing over the intersection closure}

Because bounds from corner relaxations can be significantly different, instead of relying on a single basis, it may be advantageous to consider the intersection of the corner polyhedra associated to all bases.

We now study problem $(\operatorname{int}(\mathcal{B}))$. By Theorem 3.5, $(\operatorname{int}(\mathcal{B}))$ can be expressed as

$$
\begin{array}{rlr}
z_{\operatorname{int}(\mathcal{B})}=\max & \sum_{i=1}^{n} x_{i} & \\
\text { s.t. } & x_{i}+x_{j}+y_{i j}=1 & \\
& y_{i j} \geq 0 & \forall(i, j) \in E \\
& \sum_{(i, j) \in C} y_{i j} \geq 1 & \forall C \in \mathcal{C} .
\end{array}
$$

Proposition 6.1. $z_{\text {int }(\mathcal{B})} \geq \frac{n}{3}$.

Proof. Consider vector $x_{i}^{\prime}=\frac{1}{3} \forall i \in V, y_{i j}=\frac{1}{3} \forall(i, j) \in E$. We want to prove feasibility of $x^{\prime}$. For every induced odd cycle $C \in \mathcal{C}$, denote by $l(C)$ the length of the cycle. For every $C \in \mathcal{C}$ the corresponding odd cycle constraint is satisfied:

$$
\sum_{(i, j) \in C} y_{i j}=\frac{l(C)}{3} \geq 1,
$$

where the last inequality follows by $l(C) \geq 3 \forall C \in \mathcal{C}$. All the other constraints are trivially satisfied by $x^{\prime}$, and this implies that $\sum_{i=1}^{n} x_{i}^{\prime}=\frac{n}{3}$ is a lower bound for $z_{\text {int }(\mathcal{B})}$. 
We now state a sufficient condition for $z_{\operatorname{int}(\mathcal{B})}$ to be $\frac{n}{3}$.

Theorem 6.1. Assume that there exists a partition of $V$ into cliques of size at least 3. Then $z_{\text {int }(\mathcal{B})}=\frac{n}{3}$.

Proof. Assume $G(V, E)$ can be partitioned in $h$ cliques $\left\{Q_{i}\right\}, i=\{1, \ldots, h\}$. Denote by $V\left(Q_{i}\right)$ the set of vertices in $Q_{i}$ and define the size of every clique as $s\left(Q_{i}\right)=\left|V\left(Q_{i}\right)\right|$. Note that every clique $Q_{i}$ of size at least 3 contains exactly $\left(\begin{array}{c}s\left(Q_{i}\right) \\ 3\end{array}\right)$ triangles, and each vertex is in $\left(\begin{array}{c}s\left(Q_{i}\right)-1 \\ 2\end{array}\right)$ triangles. Remark also that every odd cycle inequality of type

$$
\sum_{(i, j) \in C} y_{i j} \geq 1, \quad C \in \mathcal{C},
$$

can be rewritten in term of the $x$ variables as

$$
\sum_{i \in C} x_{i} \leq \frac{l(C)-1}{2}, \quad C \in \mathcal{C} .
$$

Consequently, for each $i \in\{1, \ldots, h\}$, summing up all triangle inequalities on clique $Q_{i}$ yields the valid inequality

$$
\left(\begin{array}{c}
s\left(Q_{i}\right)-1 \\
2
\end{array}\right) \sum_{i \in V\left(Q_{i}\right)} x_{i} \leq\left(\begin{array}{c}
s\left(Q_{i}\right) \\
3
\end{array}\right),
$$

which implies

$$
\sum_{i \in V\left(Q_{i}\right)} x_{i} \leq \frac{\left(\begin{array}{c}
s\left(Q_{i}\right) \\
3
\end{array}\right)}{\left(\begin{array}{c}
s\left(Q_{i}\right)-1 \\
2
\end{array}\right)}=\frac{s\left(Q_{i}\right)}{3} .
$$

Summing up inequalities (15) over the cliques in the partition, we get

$$
\sum_{i=1}^{n} x_{i} \leq \frac{1}{3} \sum_{i=1}^{h} s\left(Q_{i}\right)=\frac{n}{3} .
$$

By Proposition $(6.1), z_{\text {int }(\mathcal{B})} \geq \frac{n}{3}$. The two results imply $z_{\text {int }(\mathcal{B})}=\frac{n}{3}$.

Theorems 4.4, 5.3 and 6.1 imply Theorem 2.3. 


\section{Optimizing over the strict corner relaxation}

Assume that $x_{i}^{*}=\frac{1}{2} \quad \forall i=1, \ldots, n$ is an optimal solution to (LP). If $m>n$ there are many optimal bases associated with $x^{*}$. Let $B$ be one of these bases. In this section we study the strict corner relaxation of (STAB), obtained by relaxing nonnegativity of the strictly positive basic variables. The strict corner is a tighter relaxation than the corner relaxation, because the latter relaxes nonnegativity of all the basic variables, i.e., also degenerate basic variables. Note that the strict corner relaxation does not depend on the choice of $B$, since all degenerate bases associated with $x^{*}$ have the same nondegenerate basic variables.

Observe that all the edge constraints are tight at $x^{*}$. Therefore, problem $\left(\operatorname{strict}\left(x^{*}\right)\right)$ reads:

$$
\begin{array}{lll}
\max & \sum_{i=1}^{n} x_{i} & \\
\text { s.t. } & x_{i}+x_{j}+y_{i j}=1 & \forall(i, j) \in E \\
& y_{i j} \geq 0 & \forall(i, j) \in E \\
& x_{i} \in \mathbb{Z}^{n} & \forall i \in V .
\end{array}
$$

The main result of this section consists in showing that STAB and $\operatorname{strict}\left(x^{*}\right)$ have the same optimal value.

Theorem 7.1. If $x_{i}^{*}=\frac{1}{2} \forall i=1, \ldots, n$ is the optimum of $(L P), z_{S T A B}=$ $z_{\text {strict }\left(x^{*}\right)}$.

Proof. $z_{\mathrm{STAB}} \leq z_{\text {strict }\left(x^{*}\right)}$ because $(\mathrm{STR})$ is a relaxation of (STAB). By Lemma $4.1(i)$, (STR) has an optimal solution that is 0-1. This solution is feasible for $(\mathrm{STAB})$. Therefore $z_{\mathrm{STAB}} \geq z_{\text {strict }\left(x^{*}\right)}$.

Together with Theorem 4.4, this implies Theorem 2.2.

Even though $z_{\text {strict }\left(x^{*}\right)}=z_{\mathrm{STAB}}$, optimal solutions to (STR) are not always feasible for (STAB) when (LP) has alternate optimal solutions. However, when $x^{*}$ is the unique optimal solution to (STAB), the following holds.

Theorem 7.2. Suppose that $x_{i}^{*}=\frac{1}{2} \quad \forall i=1, \ldots, n$ is the unique optimal solution to (LP). Then the optimal solution to (STR) is 0-1.

Proof. Follows immediately from Lemma 4.1 (ii). 


\section{Strengthening the LP relaxation with the description of the cor- ner polyhedron}

In this section we study the strength of $(\operatorname{LP} \cap \operatorname{corner}(B))$ for an optimal basis $B$ of (LP) associated to $x_{i}^{*}=\frac{1}{2} \forall i=1, \ldots, n$.

Theorem 8.1. Given graph $G(V, E)$, let $B$ be an optimal basis of (LP) associated to $x_{i}^{*}=\frac{1}{2} \forall i=1, \ldots, n$. Suppose that $B$ is composed by $k$ 1-tree components $C_{i}\left(V_{i}, E_{i}\right), i=1, \ldots, k$. Then $\frac{n-k}{2} \leq z_{L P \cap \operatorname{corner}(B)}$. If $G$ is a clique, $z_{L P \cap \operatorname{corner}(B)}=\frac{n-k}{2}$.

Proof. We start by proving $\frac{n-k}{2} \leq z_{\mathrm{LP} \cap \operatorname{corner}(B)}$. By Theorem 3.4, the intersection of (LP) and corner $(B)$ is given by (LP) plus the odd cycle inequalities of (7). Therefore we can express problem (LP $\cap \operatorname{corner}(B))$ on $G$ in term of the $x$ variables as:

$$
\begin{array}{rlr}
z_{\mathrm{LP} \cap \operatorname{corner}(B)}=\max & \sum_{j=1}^{n} x_{j} & \\
\text { s.t. } & x_{i}+x_{j} \leq 1 & \forall(i, j) \in E \\
& x_{j} \geq 0 & \forall j \in V \\
& \sum_{j \in \kappa\left(C_{i}\right)} x_{j} \leq \frac{\left|\kappa\left(C_{i}\right)\right|-1}{2} & i=1, \ldots, k .
\end{array}
$$

Observe that problem

$$
\begin{array}{rlr}
z_{\text {clique }}=\max & \sum_{j=1}^{n} x_{j} & \\
\text { s.t. } & x_{i}+x_{j} \leq 1 & \forall(i, j) \in E \\
& x_{i}+x_{j} \leq 1 & \forall(i, j) \notin E \\
& x_{j} \geq 0 & \forall j \in V \\
& \sum_{j \in \kappa\left(C_{i}\right)} x_{j} \leq \frac{\left|\kappa\left(C_{i}\right)\right|-1}{2} & i=1, \ldots, k,
\end{array}
$$

is obtained from (16) by adding constraints relative to the edges of the complement graph. This implies $z_{\text {clique }} \leq z_{\mathrm{LP} \cap \operatorname{corner}(B)}$. Remark also that, letting

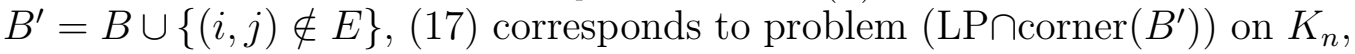
the clique defined on the vertex set $V$. This is because $N^{\prime}=N$ indexes the same 1-tree components $C_{i}, i=1, \ldots, k$. We first show $z_{\text {clique }} \leq \frac{n-k}{2}$. Partition $V$ into subsets $K=\left\{j \in V: \exists i \in\{1, \ldots, k\}\right.$ with $\left.j \in \kappa\left(C_{i}\right)\right\}$ and $V \backslash K$. The objective function of (17) can be rewritten as $\sum_{j \in K} x_{j}+\sum_{j \in V \backslash K} x_{j}$. Summing up constraints $x_{i}+x_{j} \leq 1$ for all edges of $K_{n}$ with both ends in 
$V \backslash K$ we obtain $\sum_{j \in V \backslash K} x_{j} \leq \frac{|V \backslash K|}{2}$. Similarly, the odd cycle inequalities imply

$$
\sum_{j \in K} x_{j}=\sum_{i=1}^{k} \sum_{j \in \kappa\left(C_{i}\right)} x_{j} \leq \sum_{i=1}^{k} \frac{\left|\kappa\left(C_{i}\right)\right|-1}{2}=\frac{|K|-k}{2} .
$$

Therefore,

$$
\sum_{j=1}^{n} x_{j} \leq \frac{|V \backslash K|+|K|-k}{2}=\frac{n-k}{2} .
$$

It remains to prove that a feasible solution of (17) with value $\frac{n-k}{2}$ exists. Such a solution can be easily constructed by arbitrarily choosing one vertex for each odd cycle $\kappa\left(C_{i}\right) i=1, \ldots, k$ and assigning 0 to the corresponding $x$ variables, while setting all remaining $x$ variables to $\frac{1}{2}$.

The second statement follows directly from the fact that, when $G$ is itself a clique, problems (16) and (17) coincide and $B^{\prime}=B$.

Theorem 8.2. Given an optimal basis $B$ associated to $x_{i}^{*}=\frac{1}{2} \forall i=1, \ldots, n$, the difference between $z_{\text {corner(B) }}$ and $z_{L P \cap \operatorname{corner}(B)}$ can be at most $n / 8$, and there are graphs for which this bound is tight.

Proof. By Theorem 5.1, $z_{\text {corner }(B)}=\frac{n-k_{o}}{2}$. By Theorem 8.1, $z_{\mathrm{LP} \cap \operatorname{corner}(B)} \geq$ $\frac{n-k}{2}$. It follows that the greatest gap between $z_{\text {corner }(B)}$ and $z_{\mathrm{LP} \cap \operatorname{corner}(B)}$ can occur when $\left(k-k_{o}\right)$ is maximized. Because the maximum number of even 1-tree components in a basis is at most $\left\lfloor\frac{n}{4}\right\rfloor$ the theorem follows. For a clique $K_{n}$ with $n$ multiple of 4 , we can find a basis with exactly $\frac{n}{4}$ even 1 -tree components. In this case, by Theorem 8.1, $z_{\text {corner }(B)}-z_{\mathrm{LP} \cap \operatorname{corner}(B)}=\frac{n}{8}$.

By Theorems 4.4 and 8.2, Theorem 2.4 is proven.

\section{Acknowledgements}

We thank the referees for their extremely valuable comments.

\section{References}

[1] R. E. Gomory, An algorithm for integer solutions to linear programs, in: P. Wolfe (Ed.), Recent Advances in Mathematical Programming, McGraw-Hill, New York, 1963, pp. 269-302. 
[2] G. Nemhauser, L. Wolsey, A recursive procedure for generating all cuts for 0-1 mixed integer programs, Mathematical Programming 46 (1990) $379-390$.

[3] R. E. Gomory, Some polyhedra related to combinatorial problems, Linear Algebra and its Applications 2 (1969) 451-558.

[4] M. Fischetti, M. Monaci, How tight is the corner relaxation?, Discrete Optimizaton 5 (2008) 262-269.

[5] G. R. Grimmett, An exact threshold theorem for random graphs and the node-packing problem, J. Comb. Theory Ser. B 40 (1986) 187-195.

[6] G. R. Grimmett, W. R. Pulleyblank, Random near-regular graphs and the node packing problem, Operations Research Letters 4 (1985) 169 174 .

[7] W. Cook, R. Kannan, A. Schrijver, Chvátal closures for mixed integer programming problems, Mathematical Programming 47 (1990) 155-174.

[8] S. Dash, M. Goycoolea, A heuristic to generate rank-1 GMI cuts, Mathematical Programming C 2 (2010) 231-257.

[9] M. Campêlo, G. Cornuéjols, Stable sets, corner polyhedra and the Chvátal closure, Operations Research Letters 37 (2009) 375-378.

[10] M. L. Balinski, On maximum matching, minimum covering and their connections, in: H. Kuhn (Ed.), Proceedings of the Princeton Symposium on Mathematical Programming, Princeton University Press, Princeton, N.J, 1970, pp. 303-312.

[11] G. L. Nemhauser, L. E. Trotter, Vertex packings: Structural properties and algorithms, Mathematical Programming 8 (1975) 232-248.

[12] M. W. Padberg, On the facial structure of set packing polyhedra, Mathematical Programming 5 (1973) 199-215.

[13] M. Campêlo, G. Cornuéjols, Stable sets, corner polyhedra and the Chvátal closure, 2009. Extended Version available on http://integer.tepper.cmu.edu/. 
[14] B. Bollobás, Random Graphs, Cambridge University Press, Cambridge, 2001.

[15] K. Corrádi, A. Hajnal, On the maximal number of independent circuits in a graph, Acta Mathematica Hungarica 14 (1963) 423-439.

[16] M. Krivelevich, Triangle factors in random graphs, Journal of Combinatorics, Probability and Computing 6 (1997) 337-347. 